\title{
Underwater Acoustic Communication using Reed Solomon Block Turbo Codes Channel Coding to transmit Images and Speech
}

\author{
André Goalic ${ }^{*}$, Joël Trubuil ${ }^{*}$, Christophe Laot*, and Nicolas Beuzelin ${ }^{* *}$ \\ *TELECOM Bretagne, BP 83818, 29238 Brest Cedex 3, Brittany \\ ${ }^{* *}$ G.E.S.M.A. BP 42, 29240 Naval Brest
}

\begin{abstract}
In 1997 GESMA (Groupe d'Etudes Sous-Marines de l'Atlantique), in collaboration with TELECOM Bretagne, launched a new project, called TRIDENT. First objectives were to develop a multiple rate underwater acoustic link for images, text and data transmission. This link was designed to provide a wireless communication to AUVs. Since 2006, the platform was extended to low bit rate speech transmission using a MELP coder working at 2400 bps. Such a rate allows to use lower carrier frequencies, able to reach longer ranges in the case of speech transmissions. More recently two channel coding options were added to the TRIDENT platform. Convolutionnal codes (CC) and Reed Solomon (RS) block codes were then checked. These simple codes were not able to improve significantly the Bit Error Rate (BER) at the channel decoding output. That is why, GESMA decided to introduce the turbo codes options and more precisely the Reed Solomon Block Turbo Codes (RS BTC) to enhance the channel decoding efficiency. The data transmitted are interleaved and frame recovery is performed in reception. After system validation in static condition, in Brest Penfeld river (November 2009), sea trials have been recently conducted in Brest bay (March 22 to 26, 2010) in moving condition. This paper presents the different possibilities offered by the platform for images and speech transmissions and the RS BTC channel coding options.
\end{abstract}

Keywords-Underwater acoustic communication, BER, speech coding, synchronization, Reed Solomon Block Turbo Codes (RS BTC)

\section{I.INTRODUCTION}

First objectives of the TRIDENT project (TRansmission d'Images et de Données EN Temps réel) were to equip Autonomous Underwater Vehicles (AUVs) with acoustic communications. This system had to be able to transmit continuous information such as images, data and text. The TRIDENT system (Fig. 1) [1] can use four carrier frequencies (11.2, 17.5, 20.0 and $34 \mathrm{kHz}$ ) with a QPSK modulation (Quadrature Phase Shift Keying) and different bit rates, ranging from 2.8 to $23.3 \mathrm{kbps}$. Multipath propagation, Doppler effect and noise bring a lot of perturbation on underwater acoustic communication, such as time dispersion and a variability to the received signal. It is to note that carrier frequencies and available bandwidths are much lower than communication channels ones. A blind spatio-temporal equalizer [2] is used to reduce these various perturbations. GESMA also aimed to increase the link's reliability with the objective to lower the BER from $10^{-2}$ to $10^{-4}$. In order to do so, a channel coding was integrated to the system. Two kinds of error correcting schemes had been tested including Convolutional Codes (CC) and Reed Solomon (RS) block code [3][4]. These two channel coding schemes have low code rates (CC: 0.5 and RS: 0.7 ) and do not bring us enough correction. To improve the symbol correction and obtain a higher code rate, Reed Solomon Block Turbo Codes (RS BTC) were recently tested, in static and in moving conditions. The iterative decoding process based on the Chase-Pyndiah [5][6] algorithm uses a soft version with eight iterations. Berlekamp [7] and Chien algebraic algorithms are also used to correct symbols during the iterative process. A differential coding/decoding is used to solve the phase ambiguities, with a soft decoding option. Three different synchronization words are wisely included in the coded frame to synchronize the interleaver and recover the information frame at the receiver.

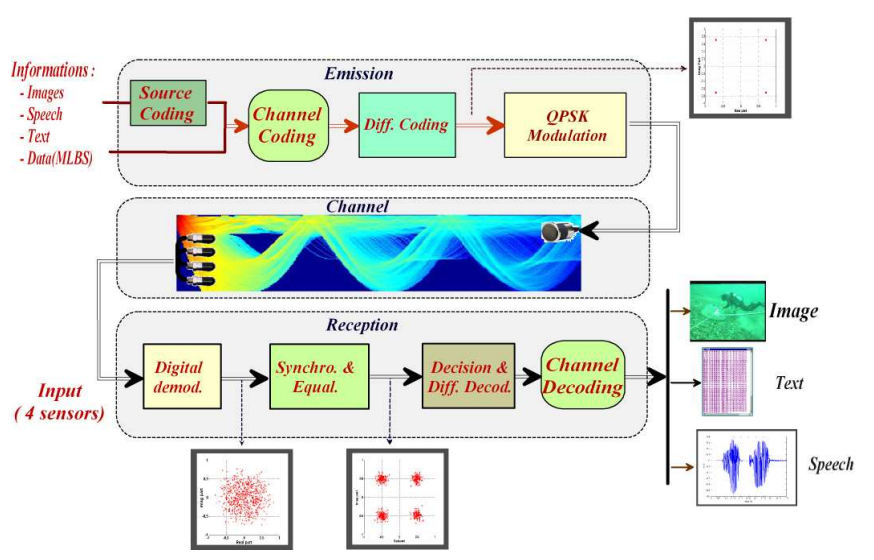

Figure 1. TRIDENT platform

This paper provides an overview of the high data rate acoustic link. Firstly, we present the TRIDENT platform and its RS Block Turbo Codes extension. Strategies for synchronizing interleaver, channel decoder and solving the phase ambiguities are also described. Then, we present the sea trials and show the acoustic communication results in the 2.8 to $14 \mathrm{kbps}$ range, applied both to images and low bit rate speech (2400 bps) transmissions. 


\section{ITERATIVE DECODING OF REED SOLOMON BLOCK TURBO CODES}

\section{A. Product codes}

The concept of product codes is a simple and efficient method to construct powerful codes with a large minimum Hamming distance, $\delta_{p}$, using conventional linear block codes [6]. Let us consider a systematic linear block code $C$ having parameters $(n, k, \delta)$ as illustrated in figure 2 . The parameters $n$ and $k$ stand for the code length and the number of information symbols respectively. The product code $P=C \times C$ is obtained by placing $k^{2}$ information bits in a matrix of $k$ rows and $k$ columns and encoding the $k$ rows and $k$ columns using the code $C$. It can be shown that all $n$ rows and all $n$ columns are codewords of $C$. Furthermore, the parameters of the resulting product code $P$ are given by $n_{p}=n^{2}, k_{p}=k^{2}, \delta_{p}=\delta^{2}$ and the code rate $R_{p}$ is given by $R_{p}=R^{2}$. Thus, it is possible to construct powerful product codes based on linear block codes such as RS codes. RS codes, working in the Galois field $G F^{q}$, are a class of linear cyclic block codes that have capabilities for multiple error detection and correction.

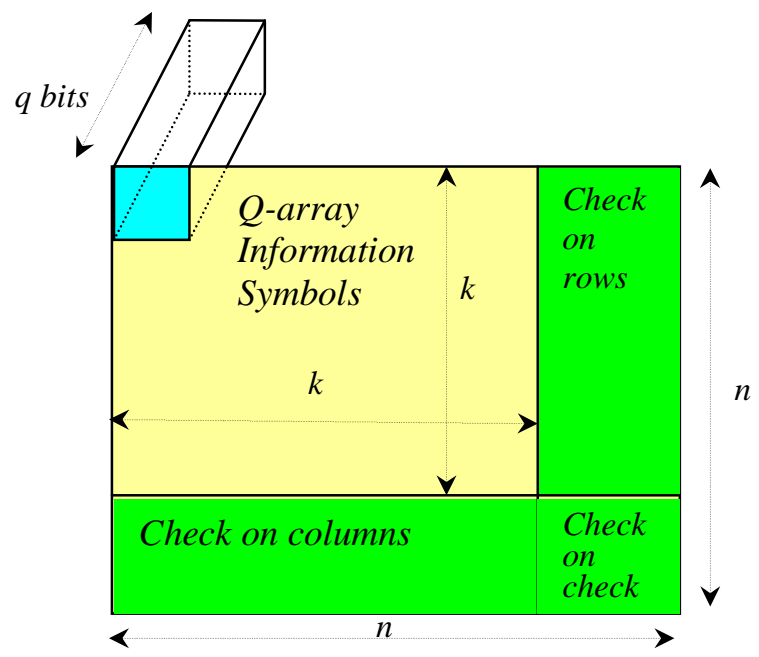

Figure 2. RS product codes with Q-ary symbol concatenation

\section{B. Iterative decoding of product codes}

Product code decoding involves sequentially decoding rows and columns using a Single Input Single Output (SISO) decoding algorithm (fig. 3). The block turbo decoding process repeats this soft decoding for several iterations. Each decoding process computes soft information $\mathbf{R}_{(i t+1)}$ from the channel received information $\mathbf{R}$ and the information computed in the previous half iteration, $\mathbf{R}^{\prime}$ (it), as shown in figure 3 . The extrinsic information $\mathbf{W}_{\text {(it) }}$ is obtained by subtracting the soft input information $\mathbf{R}^{\prime}$ (it) from the soft output information $\mathbf{F}$ (it). The soft information $\mathbf{R}_{(i t+1)}^{\prime}$ is given by $\mathbf{R}_{(\mathrm{it}+1)}^{\prime}=\mathbf{R}+\mathbf{\alpha}_{(\mathrm{it})} \mathbf{W}_{\text {(it) }}$ where $\boldsymbol{\alpha}_{(\mathrm{it})}$ is a scaling factor that reduces the effect of the extrinsic information in the soft decoder during the first decoding steps.
In 1972, Chase proposed algorithms that approximate the optimum Maximum-Likelihood (ML) decoding of block codes with low computing complexity and small performance degradation. In 1994, Pyndiah et al. presented a new iterative decoding algorithm for decoding product codes, based on the iterative SISO decoding of concatenated block codes. The Chase-Pyndiah algorithm is concisely summarized below:

1. Search for the $L_{r}$ least reliable binary bits and compute the syndrome $S_{0}$ of $\mathbf{R}^{\prime}$ (it),

2. Generate $T_{v}$ test vectors obtained by inverting some of the $L r$ least reliable binary symbols,

3. Algebraic decoding of each test vector, using Berlekamp and Chien algorithms

4. For each test vector, compute the square Euclidian distance (metric) $M_{i}\left(i=0, \ldots, T_{v n-1}\right)$ between $\mathbf{R}_{(\text {(it) }}$ and the considered test vector,

5. Select the Decided Word $D w$ having the minimal distance with $\mathbf{R}^{\prime}{ }_{(i t)}$ and choose $C w$ concurrent words having the closest distance to $\mathbf{R}$ '(it),

6. Compute reliability $\mathbf{F}_{\text {(it) }}$ for each symbol of the $D w$,

7. Compute extrinsic information $\mathbf{W}_{(\mathrm{it})}=\mathbf{F}_{(\mathrm{it})}-\mathbf{R}_{\text {(it) }}$ for each symbol of the $D w$.

The algebraic decoding used by each test vector, involves different major steps. These steps depend on the product code parameters and, in particular, on the error correction power.

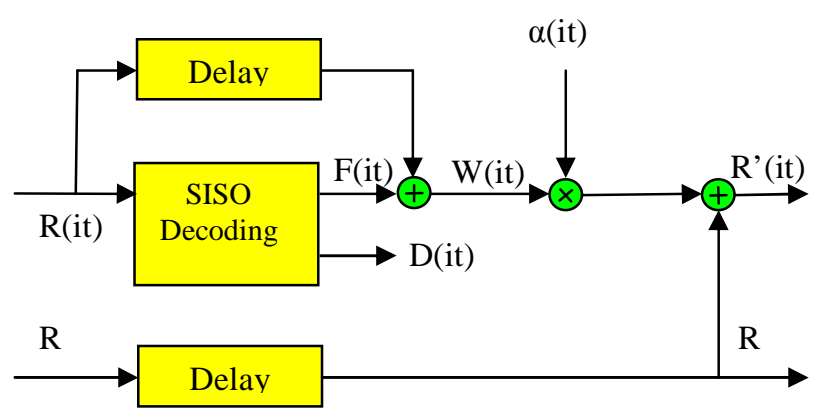

Figure 3. The iterative block turbo decoding principle

\section{FRAME SYNCHRONIZATION AND DIFFERENTIAL CODING}

Information transmitted is generally framed. In reception, synchronization words are conventionally used to synchronize both the source and channel decoders. Interleaver needs another synchronization. Several level of synchronization words lower the whole correcting rate but makes it more flexible design of different transmission system stage.

So far TRIDENT system, uses the $\operatorname{RS}^{2}(31,29,1)=\operatorname{RS}^{2}(n$ $\left.=31=2^{q}-1, k=29=n-2 t, t\right)$ BTC channel coding $(q=5, t=1$ : $\mathrm{t}$ being the correcting power). Three different synchronization 
words $S_{i}(i \in\{1,2,3\})$ are included to retrieve the frame, at the receiver. $S_{1}$ (respectively $S_{2}$ or $S_{3}$ ) is used to detect the frame start (respectively middle or end frame). The emitted coded frame, with $S_{i}$ in (respectively out) the frame, is 4805 bits in length (respectively 5084), thus the whole correcting rate is $R$ $=0.82$.

As we intrinsically have a synchronization words, it is likely that the differential coding, used to correct the phase ambiguity, becomes useless. Let us recall that differential coding leads to a $3 \mathrm{~dB}$ loss in terms of coding gain. Having a look to the frame length, the differential coding is required. Indeed, a phase jump at the frame start can generate too many errors and the expectation of a new detection is too long. Two options of differential decoding (DC) have been considered, that is to say Hard decoding (DC Hard) and Soft decoding using the Log-Likelihood Ration (DC LLR).

To improve the performance of the TRIDENT acoustic link, Reed Solomon Block Turbo Codes were chosen. Channel coding goal is to lower the bit error rate from $10^{-2}$ to $10^{-4}$ and improve images or speech synthesis quality. After synchronization and equalization efficiently realized, channel coding may add extra performance.

\section{RESULTS}

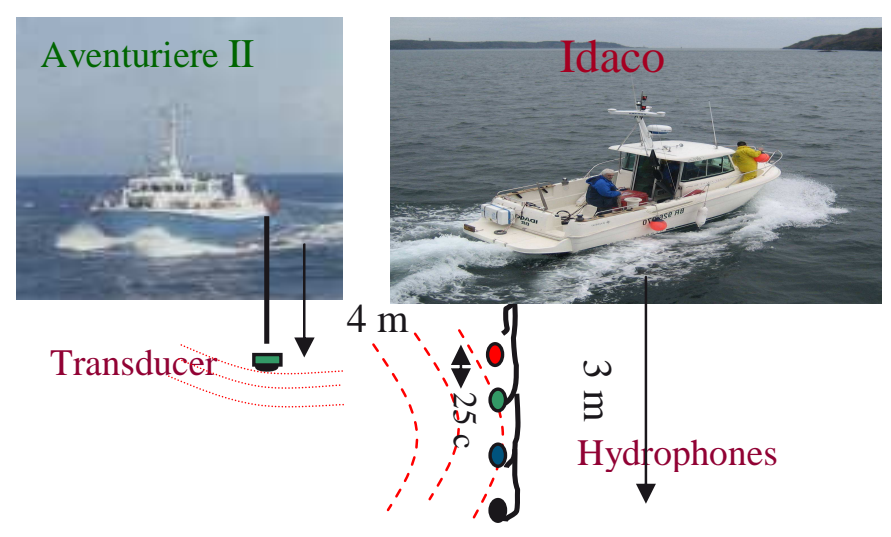

Figure 4. Sea trials configuration

Brest Bay trials took place from March 22 to 26, 2010. Brest bay is a shallow underwater acoustic channel (10-50 m depth). Two boats were used for these trials (fig. 4). The transmitter is on board the "Aventurière II". The transducer is at a $4 \mathrm{~m}$ depth. The receiver is on board the boat "Idaco". The 4 hydrophones are deployed to troll behind the boat and at $3 \mathrm{~m}$ depth of the keel. These trials were carried out in a context of rough sea. During these trails, five hours and half of signal transmitted, were recorded representing 162 sequences of 2 minutes length. Both emitter and receiver were moving (less than 4 knots) with a ranges from $300 \mathrm{~m}$ up to $3000 \mathrm{~m}$. Figure 5 represents the position of the two boats during each sequence transmission (named AIT) for tests conducted on March 24.

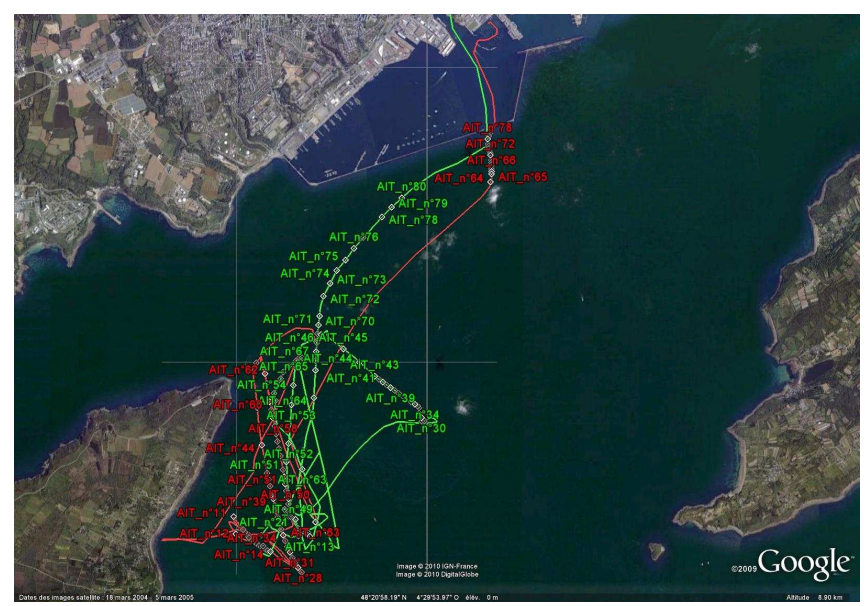

Figure 5. Brest bay sea trials: record position (march $24^{\text {th }}$ )

During these trials, 3 carrier frequencies and 4 bit rates for each, were used to transmit the information frames. Test image size represents 21171 bytes. Each transmitted frame contains approximately 600 bytes, that means we need more than 35 frames to transmit an image. Table 1 shows the frame emission time according to the bit rate used for the different results presented in this paper.

\begin{tabular}{|c|c|c|}
\hline $\begin{array}{c}\text { Carrier } \\
\text { Frequency } \\
(\mathrm{KHz})\end{array}$ & $\begin{array}{c}\text { Bit Rate } \\
(\mathrm{kbps})\end{array}$ & $\begin{array}{c}\text { Emission } \\
\text { Time } \\
(\mathrm{ms})\end{array}$ \\
\hline 17.5 & 11.6 & 410 \\
\hline 17.5 & 7.0 & 680 \\
\hline 34.0 & 8.5 & 560 \\
\hline 11.2 & 3.7 & 1290 \\
\hline
\end{tabular}

Table 1. Frame emission time

The first result, noted AIT24, show the behavior of the receiver for image transmission with a $17.5 \mathrm{kHz}$ carrier frequency and a bit rate fixed at $11.6 \mathrm{kbps}$. Transmitter and receiver speeds are around 1 knot. The distance between the transmitter and the receiver was 1000 meters. In Figure 6, the first plot show the Mean Square Error (MSE). We can note the success of the equalization process. A very good overall behavior is to be noted during this sequence. It clearly appears that the transmission was processed without any kind of difficulties. This sequence was emitted without differential coding. After decoder synchronization, 8 iterations were processed for the channel decoding. The BER at the decoder 
input $\left(\mathrm{BER}_{\text {in }}\right)$ was $1.010^{-3}$ and $1.510^{-5}$ at the decoder output. For a number of 227 frames transmitted, no frame was lost (second plot, fig. 6), 201 (88.5\%) were detected false (one or more errors) at the channel decoder input (third plot, fig. 6), and $3(1.3 \%)$ at the decoder output (fourth plot, fig. 6). During the convergence stage, frames may be lost (only one during this trial). The images was correctly decoded.
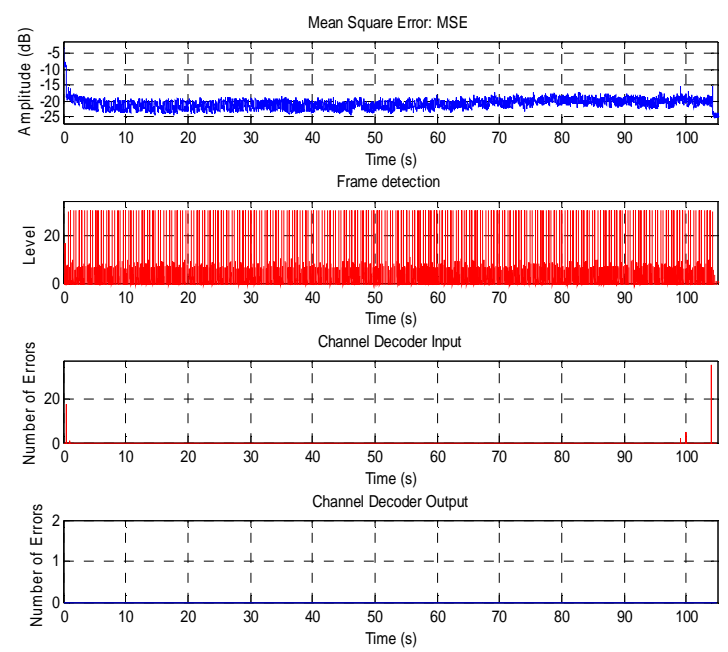

Figure 6. MSE, correlator output, decoder input and decoder output (AIT24)
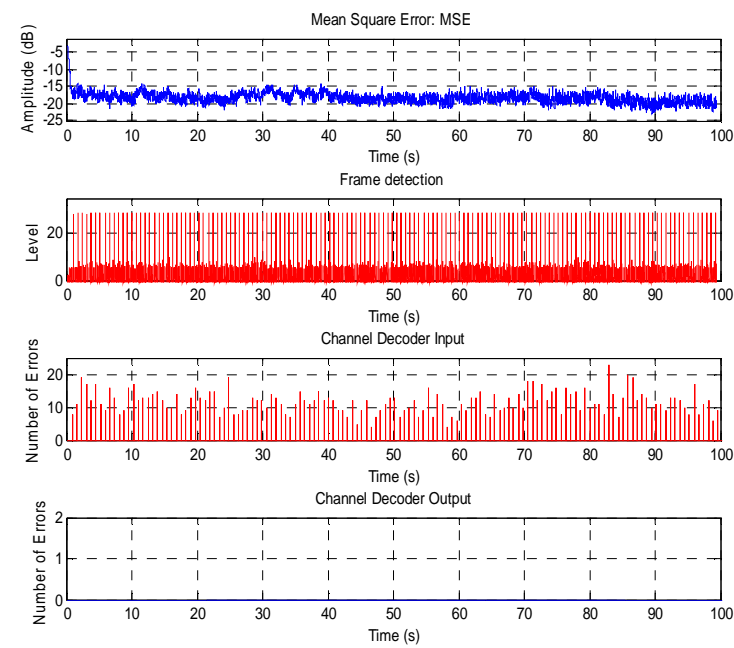

Figure 7. MSE, correlator output, decoder input and decoder output (AIT30)

The second result, noted AIT30, concern a speech signal transmission. For this trial, the carrier frequency was at 17.5 $\mathrm{kHz}$ and the bit rate fixed at $7.0 \mathrm{kbps}$ with $2.4 \mathrm{kbps}$ used for the speech signal. The distance between the transmitter and the receiver was 2500 meters. The MSE (first plot, fig. 7) also shows the equalization success. After both phase ambiguity correction and decoder synchronization, the BER at the decoder input $\left(\mathrm{BER}_{\mathrm{in}}\right)$ was $2.410^{-3}$ and the $\mathrm{BER}_{\text {out }}$ was 0.0 at the decoder output. For a number of 144 frames transmitted, no frame was lost. The Frame Error Rate Input $\left(\mathrm{FER}_{\text {in }}\right)$ was 144 $(100 \%)$ and the $\mathrm{FER}_{\text {out }}$ was $0(0.0 \%)$ at the decoder output. 8 iterations were processed for the channel decoding. The speech signal was correctly decoded (fig. 8).

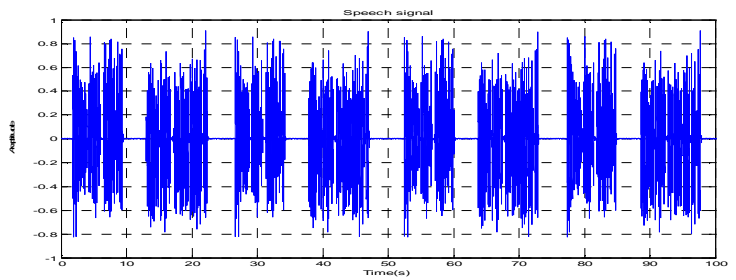

Figure 8. Speech signal (AIT30)

The third result, noted AIT151, concern images transmission. The distance between the transmitter and the receiver was 1000 meters. For this trial, the carrier frequency was at $11.2 \mathrm{kHz}$ and the bit rate fixed at $3.7 \mathrm{kbps}$. In this trial, the sequence was emitted without differential coding, so a phase jump appearing at the end of the transmission was not detected. The MSE (first plot, fig. 9) also shows the equalization success, just disturbed during the phase jump. The $\mathrm{BER}_{\text {in }}$ at the decoder input was $1.710^{-4}$ and the $\mathrm{BER}_{\text {out }} 0.0$ at the decoder output, evaluated before the phase jump. For a number of 62 frames transmitted, no frame was lost. The FER $\mathrm{F}_{\text {in }}$ was $26(100 \%)$ and the FER $_{\text {out }}$ was $0(0.0 \%)$ at the decoder output. 8 iterations were processed for the channel decoding. The images was correctly decoded before the phase jump.
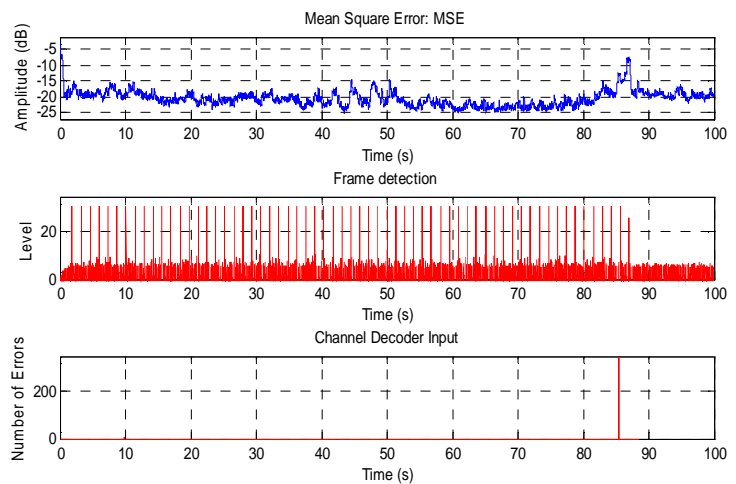

Channel Decoder Output

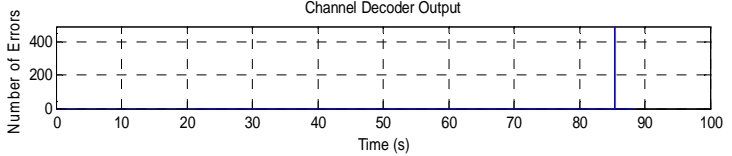

Figure 9. MSE, correlator output, decoder input and decoder output (AIT151) (undetected phase jump in the sequence) 
The last result, noted AIT105, is about images transmission. The carrier frequency was at $34.0 \mathrm{kHz}$ and the bit rate fixed at $8.5 \mathrm{kbps}$. The distance between the transmitter and the receiver was 300 meters. In this trial, the MSE (first plot, fig. 10) was corrupted at the beginning of the sequence during 12 seconds. The $\mathrm{BER}_{\text {in }}$ level for a few frames does not allow an efficient work of the channel decoding. The sequence is also corrupted in its middle (from the 47 th and the 52 th second). When the BER is not too high (BER $<1 \%$ ), the channel decoding runs correctly, otherwise the frame is indecodable. For a 176 frames transmitted, 176 were detected false (one or more errors) at the channel decoder input. At the output, 23 frames were detected false. The following statistics only concern the decodable frames: $\mathrm{BER}_{\mathrm{in}}=4.3610^{-3}, \mathrm{BER}_{\text {out }}=0.0$ and $\mathrm{FER}_{\text {in }}=152(100 \%), \mathrm{FER}_{\text {out }}=0(0.0 \%)$.

Figure 11 shows the state of an image after iteration 1, 2, 3 and 8 . The residual errors appearing in the middle of the sequence does not allow a complete recovery of the image.
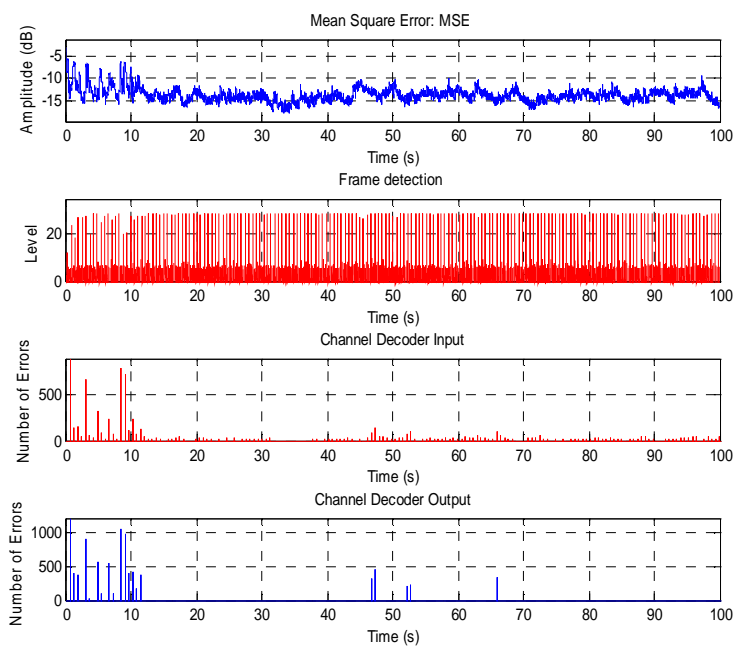

Figure 10. MSE, correlator output, decoder input and decoder output (AIT105)

\section{CONCLUSION and PERSPECTIVES}

This paper presents the extension of the TRIDENT system, developed by GESMA in collaboration with TELECOM Bretagne. This underwater acoustic link is designed to transmit different kinds of data as text, images and speech signal. A blind spatio-temporal equalizer is used to reduce different shallow underwater acoustic perturbations. To improve the underwater acoustic link performance and obtain a higher code rate, Reed Solomon Block Turbo Codes (RS BTC) were introduced and tested in real conditions, with the aim to decrease the BER from $10^{-2}$ to $10^{-4}$. A differential coding has been used to solve the phase ambiguities. First sea trials of real time transmission of speech signal and images, using channel turbo coding, were carried out in stationary condition, in Brest Penfeld river, (November, 2009). 100 experiments were carried out on this site with success. In March 2010, sea trials were carried out in the Brest bay, in moving conditions and rough sea. More than 5 hours of transmitted signal were recorded. A large number of sequences are successfully decoded at different distances 300, 1000 and 2500 meters in moving condition, using three carrier frequencies and different bit rates from 3.7 to $11.6 \mathrm{kbps}$. These sequences show a good behavior of the channel coding/decoding when synchronization and equalization work correctly. However, given test conditions at sea, frames remained indecodable by the channel decoder. In order to enhance the system robustness, next works will be carried out to improve both the Doppler effect and the timing recovery.

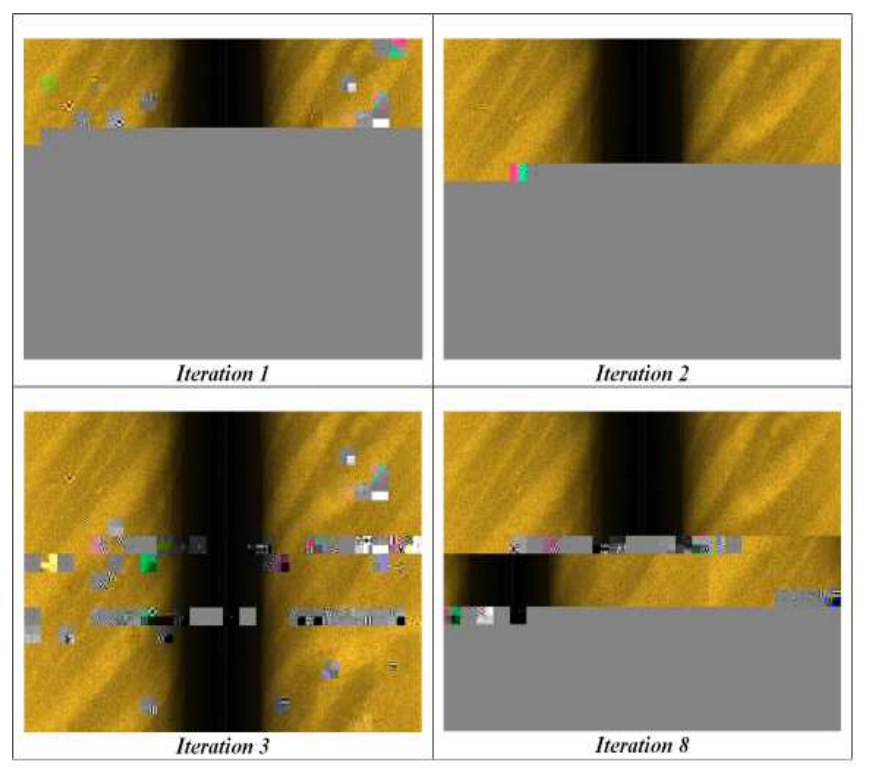

Figure 11. Image 2/4 (AIT105)

\section{REFERENCES}

[1] J. Trubuil, G. Lapierre, T. Gall, J. Labat, "Real-time high data rate acoustic link based on spatio temporal blind equalization: the TRIDENT acoustic system," in Proc. OCEANS 2002, Biloxi, Vol.4, pp2438-2443.

[2] J. Labat and C. Laot, "Blind adaptive multiple input decision feedback equalizer with a self optimized configuration", IEEE trans on Comm, Vol. 49, $\mathrm{N}^{\circ} 4$, April 2001.

[3] A. Goalic, J.Trubuil and N. Beuzelin "Channel coding for underwater acoustic communication system”, Oceans 2006, September 18-21, Boston, Ma, USA.

[4] J.Trubuil, A. Goalic and N. Beuzelin, "Synchronization and channel coding in shallow water acoustic communication", Oceans 2008, September 15-18, Québec, Canada..

[5] R, Pyndiah, A, Glavieux, A, Picart and S. Jacq, "Near optimum decoding of product codes", in Proc. Of IEEE Globecom '94, vol. 1/3, Nov.-Dec. 1994, pp.339-343

[6] D. Chase, "A class of algorithms for decoding block codes with channel measurement information", IEEE trans. Inform. Theory, vol IT-8, Jan. 1972.

[7] E. R. Berlekamp, "Algebraic coding Theory", McGraw-Hill Book Company, New York, 1968 